\title{
Quercetin protects against ox-LDL-induced injury via regulation of ABCAl, LXR- $\alpha$ and PCSK9 in RAW264.7 macrophages
}

\author{
SHANSHAN LI*, HUI CAO*, DINGZHU SHEN, QINGLING JIA, CHUAN CHEN and SAN LI XING \\ Shanghai Geriatrics Institute of Chinese Medicine, Shanghai 200031, P.R. China
}

Received December 3, 2017; Accepted May 4, 2018

DOI: $10.3892 / \mathrm{mmr} .2018 .9048$

\begin{abstract}
Quercetin is a flavonoid that has anti-inflammatory, anti-oxidant and lipid metabolic effects. It has also been reported to reduce the risk of cardiovascular disease. The present study measured the effects of quercetin on the expression of ATP-binding cassette transporter 1 (ABCAl), ATP-binding cassette sub-family $\mathrm{G}$ member 1 (ABCG1), liver $\mathrm{X}$ receptor- $\alpha$ (LXR- $\alpha$ ), proprotein convertase subtilisin/kexin type 9 (PCSK9), p53, p21 and p16 induced by oxidized low density lipoprotein (ox-LDL). RAW264.7 macrophages were exposed to ox-LDL with or without $20 \mu \mathrm{mol} / 1$ quercetin and cell proliferation and senescence were quantified using $\beta$-gal staining. Superoxide dismutase (SOD), malondialdehyde (MDA) and lipid droplets were measured in the cytoplasm using oil red staining, while intracellular and total cholesterol (TC) were measured using filipin staining and a TC kit. Immunofluorescent studies and western blot analysis were performed to quantify the expression of ABCA1, ABCG1, LXR- $\alpha$, PCSK9, p53, p21 and p16. Quercetin increased RAW264.7 cell viability and reduced lipid accumulation, senescence, lipid droplets, intracellular cholesterol and TC. It was concluded that quercetin inhibits ox-LDL-induced lipid droplets in RAW264.7 cells by upregulation of ABCA1, ABCG1, LXR- $\alpha$ and downregulation of PCSK9, p53, p21 and p16.
\end{abstract}

\section{Introduction}

Atherosclerosis (AS) is a common consequence of cardiovascular disease that includes abnormal lipid metabolism (1) and foam cell formation, which indicate early AS (2). Thus, blocking foam cell formation may be a key to reducing AS (3).

Correspondence to: Dr Dingzhu Shen, Shanghai Geriatrics Institute of Chinese Medicine, 365 South Xiangyang Road, Xuhui, Shanghai 200031, P.R. China

E-mail: 13818279131@163.com

*Contributed equally

Key words: quercetin, macrophage, ATP-binding cassette transporter 1, liver $\mathrm{X}$ receptor- $\alpha$, proprotein convertase subtilisin/kexin type 9
ATP-binding cassette transporter A1 (ABCA1), a critical protein for reverse cholesterol transport (RCT), promotes macrophage cholesterol efflux and prevents foam cell formation. ATP-binding cassette sub-family G member (ABCG1) is an active lipid transporter and a member of the ABC family. Both of them highly expressed in many tissues and can be activated by the liver X receptors (LXRs) (4). It contributes to RCT. Proprotein convertase subtilisin/kexin type 9 (PCSK9) is a lipid regulatory protein involved in lipid metabolism and apoptosis (5). Previous studies confirm that PCSK9 is upregulated in macrophages, causing an inflammatory response and cholesterol accumulation via inhibition of RCT (6). However, inhibition of PCSK9 can intervene in AS. Also, PCSK9 inhibitors can promote macrophage cholesterol efflux via upregulation of ABCA1 (7).

Quercetin is a flavonoid reported to have anti-inflammatory, anti-oxidant, and lipid metabolic functions (8). It may prevent AS by regulating lipid metabolism, enhancing expression of ABCA1 in RAW264.7 cells, promoting macrophage cholesterol efflux and blocking foam cell formation (9). Studies suggested that quercetin inhibited PCSK9 expression in hepatocytes and promoted macrophage cholesterol efflux (10).

Thus, we assessed whether quercetin prevented ox-LDL-induced lipid deposition in macrophages via increased expression of ABCA1 and LXR- $\alpha$ and decreased expression of PCSK9. RAW264.7 macrophages were induced with ox-LDL to create a cell injury model, and cell viability was assessed. Lipid deposition and expression of ABCA1, ABCG1, LXR- $\alpha$, PCSK9, P53, P21 and P16 were also measured.

\section{Materials and methods}

Materials. Mouse macrophage RAW264.7 cells were purchased from the Shanghai Institute of Biochemistry and Cell, Shanghai, China. Dulbecco's modified eagle medium (DMEM) was purchased from Gibco (Thermo Fisher Scientific, Inc., Waltham, MA, USA), FBS was purchased from Invitrogen (Thermo Fisher Scientific, Inc.) Quercetin was purchased from Shanghai Yuanye Bio-Technology Co., Ltd. (Shanghai, China). Oxidized low density lipoprotein (ox-LDL) was purchased from Shanghai Yuanye Bio-Technology Co., Ltd. The protein ladder was from Thermo Fisher Scientific, Inc. We used anti-ABCA1 (Thermo Fisher Scientific, Inc.), anti-ABCG1 anti-PCSK9, anti-LXR- $\alpha$, anti-P53, anti-P21, anti-P16 antibodies were all purchased from Abcam (Cambridge, MA, USA), anti- $\beta$-actin 
(Cell Signaling Technology, Inc., Danvers, MA, USA), goat anti-rabbit $\operatorname{IgG}(\mathrm{H}+\mathrm{L})$ secondary antibody, goat anti-mouse $\operatorname{IgG}(\mathrm{H}+\mathrm{L})$ secondary antibody (both LI-COR, Lincoln, NE, USA); FITC-labeled goat anti-mouse IgG $(\mathrm{H}+\mathrm{L})$ (Shanghai Beyotime, Shanghai, China), and FITC-labeled goat anti-rabbit $\mathrm{IgG}(\mathrm{H}+\mathrm{L})$ (A0562; Shanghai Beyotime).

A CCK8 kit was purchased from Shanghai Beyotime. An enhanced BCA protein assay kit was used as was an SDS-PAGE preparation kit (both from Shanghai Beyotime). We used an oil red O staining kit Shanghai Yeasen (Shanghai, China) and a senescence $\beta$-galactosidase ( $\beta$-gal) staining kit (Shanghai Beyotime). SOD and MDA were measured using assay kit (Nanjing Jiancheng Bioengineering Institute, Nanjing, China). A Filipin staining kit was purchased from Shanghai Genmed (Shanghai, China), and DAPI staining solution was purchased from Shanghai Beyotime.

Macrophage cell culture. RAW264.7 cells were cultured in DMEM medium containing 10\% FBS in a humidified atmosphere at $37^{\circ} \mathrm{C}, 5 \% \mathrm{CO}_{2}$. Then the cells were passaged three times. When the cells were confluent, they were randomized into four groups: Control (carrier medium), ox-LDL treatment, ox-LDL + quercetin treatment, and quercetin treatment using at least three replicates for each group. Cells were cultured with ox-LDL for $24 \mathrm{~h}$ at $100 \mathrm{mg} / \mathrm{l}$ or quercetin $(20 \mu \mathrm{mol} / \mathrm{l})$ or an ox-LDL/quercetin co-culture. Subsequently, cells were harvested and extracted for analysis.

Cell proliferation assay. RAW264.7 cells were seeded into 96-well plates $\left(10^{3} /\right.$ well), and $100 \mu l$ medium was added into each well. Plates were cultured for $24 \mathrm{~h}$ in an incubator containing $5 \% \mathrm{CO}_{2}$ at $37^{\circ} \mathrm{C}$. Then cells were randomized to ox-LDL $(0$, $10,50,100$, or $200 \mathrm{mg} / \mathrm{l})$, quercetin $(10,20,40$, or $60 \mu \mathrm{mol} / \mathrm{l})$, control (vehicle medium), or ox-LDL treated, ox-LDL + quercetin, or quercetin. After culture for $24 \mathrm{~h}$, cell proliferation was measured using a CCK8 kit and a microplate reader (490 nm).

Oil red $O$ and Filipin staining. RAW264.7 cells were randomized to control, ox-LDL, ox-LDL + quercetin or quercetin, as described above. Cell cultures were rinsed once with PBS and fixed with $10 \%$ (v/v) formaldehyde for $20 \mathrm{~min}$ at room temperature. After three washings with distilled water, cells were incubated with filtered $60 \%$ (v/v) isopropyl alcohol for $1 \mathrm{~min}$ and then fixed with oil red $\mathrm{O}$ solution for $15 \mathrm{~min}$ at room temperature. Cell cultures were rinsed in distilled water and counterstained with hematoxylin for $30 \mathrm{sec}$, rinsed with distilled water and sealed with glycerin gelatin. Intracellular lipid droplets were red, and nuclei were blue under a microscope. Oil red O imaging was manually performed with the $\mathrm{x} 40$ objective lens, and from the images, five areas were randomly selected for analysis using integrated optical density (IOD) and ImageJ software (National Institutes of Health, Bethesda, MD, USA) to quantify intracellular lipid droplets.

RAW264.7 cells were treated as described and washed once with PBS and then stained with Filipin according to kit instructions. Intracellular unesterified cholesterol was stained by Filipin and fluoresced blue $\left(340 \mathrm{~nm}_{\mathrm{ex}} ; 430 \mathrm{~nm}_{\mathrm{em}}\right)$.

Total cholesterol (TC) assay. RAW264.7 cells were treated as described above. TC was measured according to kit instructions. Briefly, after treatment, cells were washed once with PBS and lysed using an ultrasonic homogenizer and heated for $10 \mathrm{~min}$ at $70^{\circ} \mathrm{C}$. Then, the cells were centrifuged at $2,000 \mathrm{rpm} / \mathrm{min}$ for $5 \mathrm{~min}$ at room temperature. The supernatant was removed, and TC was measured using a microplate reader. Protein was quantified using a BCA kit, and TC was normalized to the protein content.

SOD measurement. Cells were scraped, and SOD was assayed for $10 \mathrm{~min}$. Cells were then centrifuged for $10 \mathrm{~min}$ at $1,500 \mathrm{rpm} / \mathrm{min}$ and precipitated. Buffer was added to the cells, and ultrasonic lysis was carried out. Reagent was added to the cells according to the SOD assay instructions and incubated at $37^{\circ} \mathrm{C}$ for $20 \mathrm{~min}$. Absorption was read at $450 \mathrm{~nm}$.

MDA measurement. Cells were treated as they were for the SOD assays and heated at $95^{\circ} \mathrm{C}$ for $20 \mathrm{~min}$. Reagents were prepared according to MDA kit instructions, and the pore plate was treated as instructed. The absorption was read at $530 \mathrm{~nm}$.

Immunofluorescent assay. RAW264.7 cells were randomized to control, ox-LDL, ox-LDL + quercetin, or quercetin. After cell routine treatment, cells were washed three times with PBS and then fixed in 4\% paraformaldehyde for $10 \mathrm{~min}$ at room temperature. Cells were washed three times with PBS and then saturated with $10 \%$ BSA for $30 \mathrm{~min}$. Samples were incubated with anti-ABCA1, anti-ABCG1, anti-LXR- $\alpha$, anti-PCSK9, anti-P53, anti-P21, anti-P16 overnight at $4^{\circ} \mathrm{C}$. After washing twice with PBS, samples were incubated with secondary antibody FITC-labeled goat anti-rabbit IgG (1:50) for $30 \mathrm{~min}$ at room temperature. After staining for quantification, samples were incubated with $10 \mu \mathrm{g} / \mathrm{ml}$ DAPI for $5 \mathrm{~min}$ for visualization of the cell nuclei. Finally, cells were washed three times with PBS and sealed. The average integral optical density of green fluorescence was analyzed using ImageJ software (National Institutes of Health) to reflect the relative protein expression.

Western blot assay. RAW264.7 cells were treated as described and washed three times with PBS, and cell lysates were generated using RIPA lysis buffer with PMSF. Cells were scraped and removed to a new tube. The solution was lysed on ice for $30 \mathrm{~min}$ and centrifuged at $12,000 \mathrm{rpm} / \mathrm{min}$ for $30 \mathrm{~min}$ at $4^{\circ} \mathrm{C}$. Then, the supernatant was assayed for protein with a BCA kit. The protein concentration was adjusted with RIPA lysate, and 5x loading buffer was added and boiled for $5 \mathrm{~min}$. Then, cells were stored at $-80^{\circ} \mathrm{C}$. In total, $30 \mu \mathrm{g}$ protein was resolved by SDS-PAGE and transferred to PVDF membranes, which were blocked in TBST with 5\% BSA, and incubated with anti-ABCA1, anti-ABCG1, anti-LXR- $\alpha$, anti-PCSK9, anti-P53, anti-P21, anti-P16 and anti- $\beta$-actin overnight at $4^{\circ} \mathrm{C}$. Membranes were incubated with appropriate horseradish peroxidase-conjugated secondary antibodies for $1 \mathrm{~h}$ and then washed to remove unbound antibodies. Finally, membranes were incubated with ECL HRP substrate and imaged with an ECL system. Protein bands were visualized using Image J software (National Institutes of Health), and the target protein was quantified. 

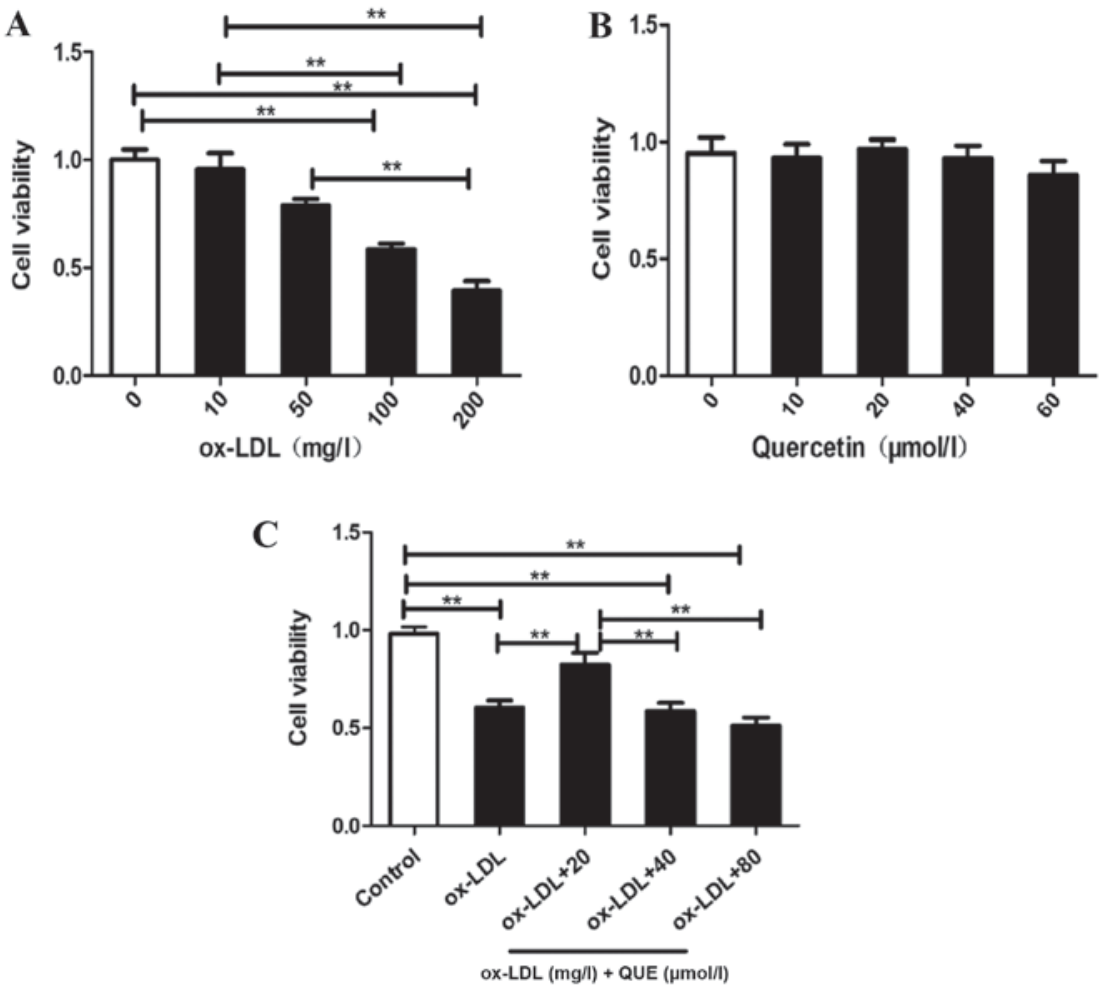

Figure 1. Quercetin and RAW264.7 macrophage cell viability. RAW264.7 cells were treated with (A) ox-LDL, (B) quercetin or (C) quercetin and ox-LDL and the cell viability was determined. Data are presented as the mean \pm standard deviation. ${ }^{* *} \mathrm{P}<0.01$. ox-LDL, oxidized low density lipoprotein; QUE, quercetin.

$\beta$-gal. Expression of $\mathrm{pH}$-dependent senescence-associated $\beta$-gal in RAW264.7 cells was analyzed using a $\beta$-gal staining kit according to the manufacturer's instructions. Briefly, RAW264.7 cells grown in a microwell plate were washed and incubated in a fixative solution. After one wash with PBS, $1 \mathrm{ml}$ $\beta$-gal staining solution was added to each well. The plate was sealed with Parafilm to prevent evaporation and incubated at room temperature for $15 \mathrm{~min}$. Then the fixative solution was removed, and cells were washed with PBS 3 times. Finally, cells were incubated with special dyeing fluid at $37^{\circ} \mathrm{C}$ overnight. Cells were examined under a light microscope. Cells were counted, and those that were blue were considered positive.

Statistical analyses. Data are presented as the mean + standard deviation. An unpaired Student's t-test was used to compare two independent groups and one-way ANOVA with Tukey HSD post hoc test was used to compare multiple independent groups with SPSS17.0 software. For all tests, $\mathrm{P}<0.05$ was considered to indicate a statistically significant difference.

\section{Results}

Quercetin improved RAW264.7 cells viability induced by $o x-L D L$. RAW264.7 cells viability were assessed, and Fig. 1A shows that ox-LDL reduced RAW264.7 cell viability in a concentration-dependent manner. Half of the cells were viable with $100 \mathrm{mg} / \mathrm{ml}$ ox-LDL, quercetin was not cytotoxic to RAW264.7 cells at $60 \mu \mathrm{mol} / 1$ (Fig. 1B). Fig. 1C shows that treatment of ox-LDL-induced RAW 264.7 cells with $20 \mu \mathrm{mol} / 1$ quercetin significantly improved viability. Thus, quercetin blocked ox-LDL reductions in RAW264.7 cells proliferation.

Quercetin inhibited lipid accumulation in RAW264.7 cells induced by ox-LDL. We measured macrophage lipid accumulation in RAW264.7 cells at $24 \mathrm{~h}$ and oil red staining showed that lipid droplets in the ox-LDL + quercetin group were significantly less than in the ox-LDL group $(\mathrm{P}<0.05)$. There was no significant difference between controls and ox-LDL + quercetin groups $(\mathrm{P}<0.05)$ (Fig. 2A). Intracellular free cholesterol was measured using Filipin staining and it was less in the ox-LDL + quercetin group (Fig. 2B). TC was measured and quercetin reduced ox-LDL-induced TC (Fig. 2C). Thus, quercetin inhibits lipid accumulation in RAW264.7 cells induced by ox-LDL.

Quercetin effectively regulated the expression of $A B C A l$, ABCG1, LXR- $\alpha$, PCSK9, P53, P21, P16 in RAW264.7 cells. The effects of quercetin on ABCA1, LXR- $\alpha$ and PCSK9 expression were measured and immunofluorescent data show that ox-LDL decreased expression of ABCA1, ABCG1 and LXR- $\alpha$, but increased PCSK9, P53, P21 and P16 expression. After treatment with quercetin, ABCA1, ABCG1 and LXR- $\alpha$ expression were upregulated, and expression of PCSK9, P53, P21 and P16 were downregulated (Fig. 3). We verified that quercetin modulated ABCA1, ABCG1, LXR- $\alpha$, PCSK9, P53, P21 and P16 expression by Western Blot, and data agreed with immunofluorescent results. Thus, ox-LDL decreased expression of ABCA1, ABCG1 and LXR- $\alpha$, but increased PCSK9, P53, P21and P16 expression. After treatment with quercetin, ABCA1, ABCG1 and LXR- $\alpha$ 

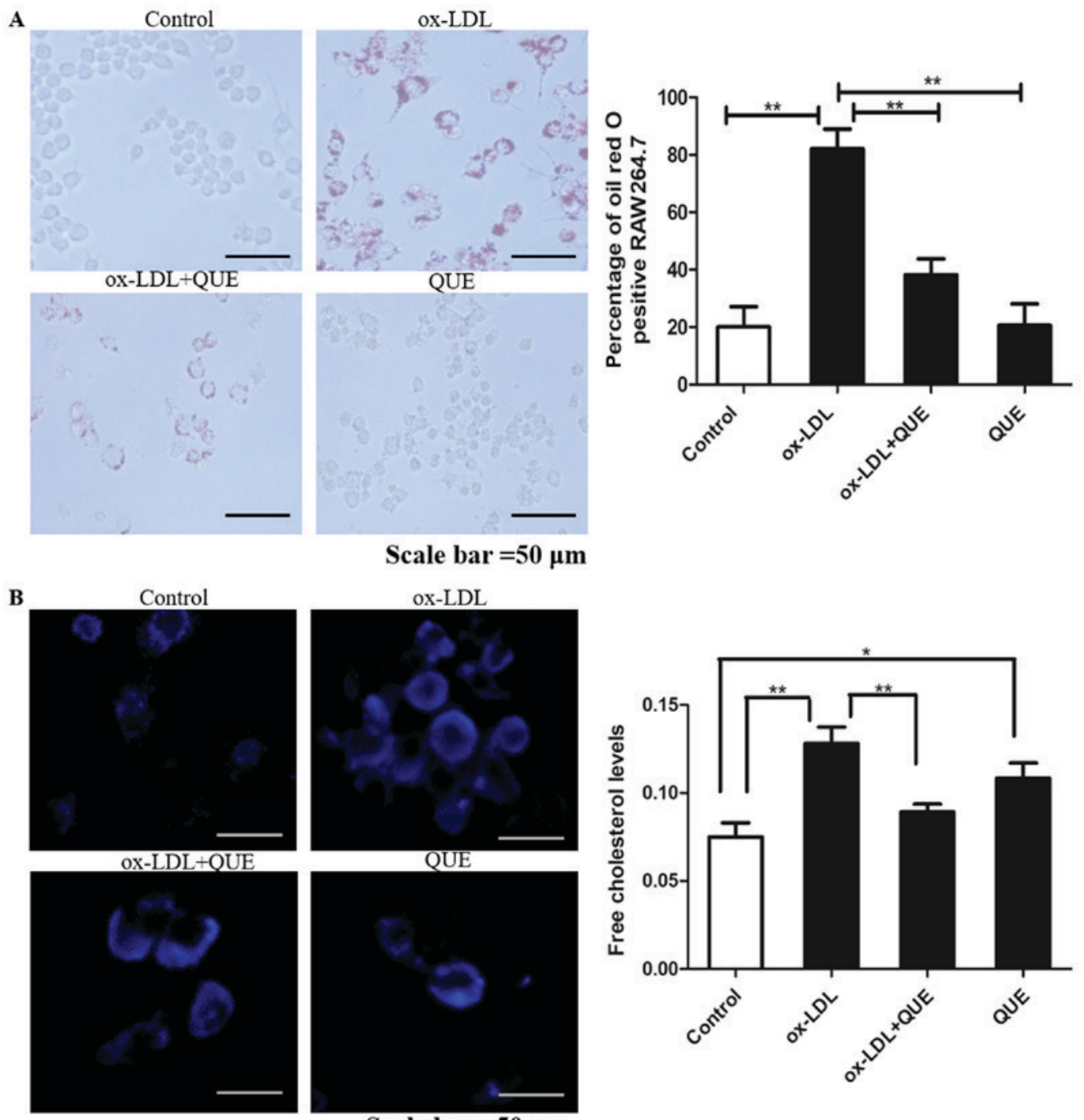

C

Scale bar $=\mathbf{5 0} \mu \mathrm{m}$

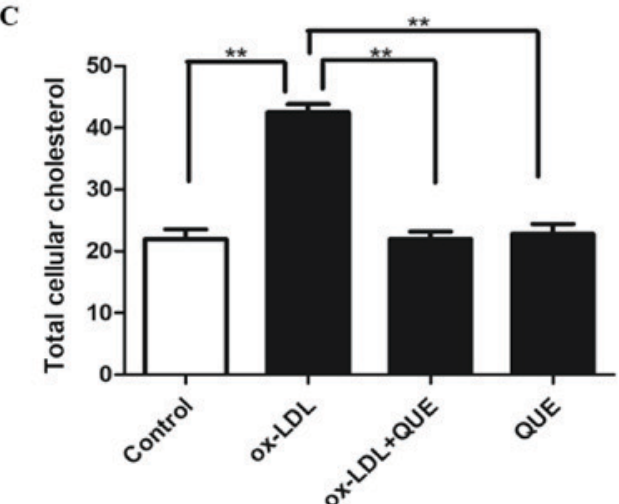

Figure 2. Quercetin and lipid accumulation in RAW264.7 cells induced by ox-LDL. (A) RAW264.7 cells were treated with quercetin and ox-LDL and the lipid accumulation was measured by oil red staining. (B) RAW264.7 cells were treated with quercetin and ox-LDL and the intracellular free cholesterol was measured using Filipin staining. (C) RAW264.7 cells were treated with quercetin and ox-LDL and the total cellular cholesterol was measured. Data are presented as the mean \pm standard deviation. ${ }^{*} \mathrm{P}<0.05,{ }^{* *} \mathrm{P}<0.01$. Ox-LDL, oxidized low density lipoprotein; QUE, quercetin.

expression was upregulated, and expression of PCSK9, P53, P21and P16 was downregulated (Fig. 4). Thus, quercetin can modify expression of ABCAl, ABCG1, LXR- $\alpha$, PCSK9, P53, P21 and P16 in RAW264.7 cells.

Quercetin slowed RAW 264.7 cells senescence. We studied whether quercetin could reduce RAW264.7 cells senescence after ox-LDL intervention using $\beta$-gal staining. Fig. 5 shows that ox-LDL accelerated cell senescence, and quercetin reduced senescent cells. There was no statistically significant difference between the ox-LDL + quercetin and quercetin groups. Data show that quercetin significantly reduced RAW 264.7 cell senescence.

Effects of quercetin on SOD activity and MDA in RAW 264.7 cells. SOD and MDA were measured after quercetin treatment 

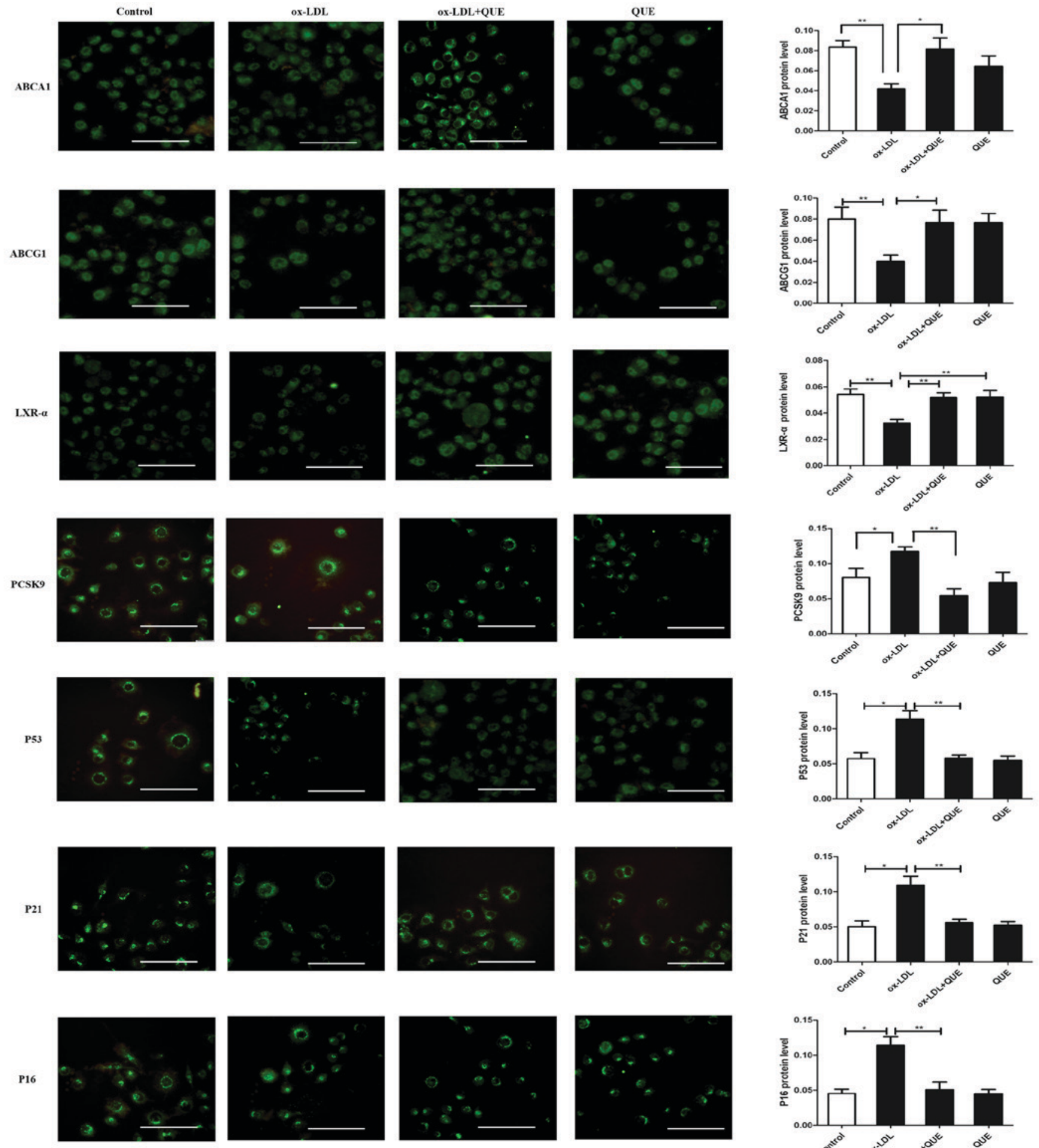

Scale bar=50 $\mu \mathrm{m}$

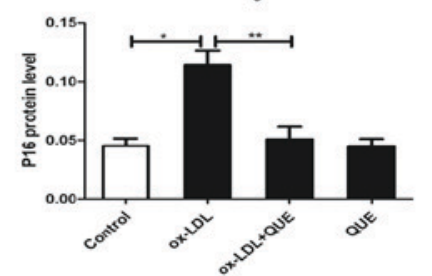

Figure 3. ABCA1, LXR- $\alpha$ and PCSK9 expression changes following quercetin treatment in ox-LDL-induced RAW264.7 cells. Immunofluorescent quantification of ABCA1, LXR- $\alpha$ and PCSK9 proteins. Data are presented as the mean \pm standard deviation. ${ }^{*} \mathrm{P}<0.05,{ }^{* * *} \mathrm{P}<0.01$. Scale bar, $50 \mu \mathrm{m}$. ABCA1, ATP-binding cassette transporter 1; LXR- $\alpha$, liver X receptor- $\alpha$; PCSK9, proprotein convertase subtilisin/kexin type 9; ox-LDL, oxidized low density lipoprotein; QUE, quercetin.

of RAW264.7 cells. Table I shows that viability in the ox-LDL group was less than other groups and this difference was statistically significant $(\mathrm{P}<0.01)$. Ox-LDL group's MDA was greater than the other groups and this was statistically significant $(\mathrm{P}<0.01)$. Thus, quercetin increased SOD activity after ox-LDL treatment and blocked the effect of quercetin on MDA.

\section{Discussion}

AS contributes to many cardiovascular and cerebrovascular diseases and lipid metabolic disorders contribute to AS. When macrophages accumulate in vessels, macrophages transform into foam cells and release intracellular cholesterol which 

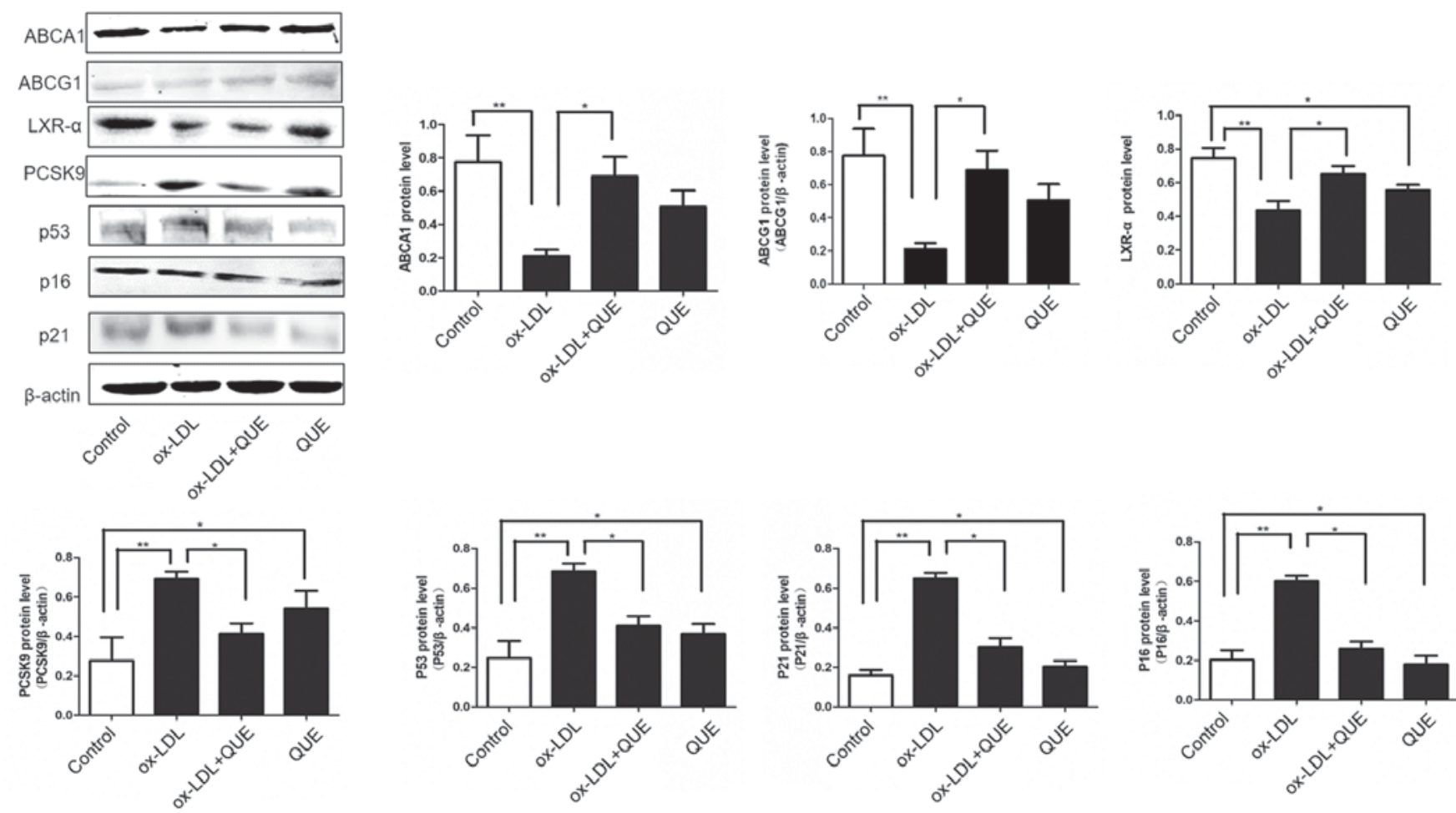

Figure 4. ABCA1, ABCG1, LXR- $\alpha$, PCSK9, p53, p21 and p16 expression changes following quercetin treatment in ox-LDL-induced RAW264.7 cells. Western blot analysis quantification of ABCA1, ABCG1, LXR- $\alpha$, PCSK9, p53, p21 and p16 proteins. Data are presented as the mean \pm standard deviation. ${ }^{*} \mathrm{P}<0.05$, ${ }^{* *} \mathrm{P}<0.01$. ABCA1, ATP-binding cassette transporter 1; ABCG1, ATP-binding cassette sub-family G member 1; LXR- $\alpha$, liver X receptor- $\alpha$; PCSK9, proprotein convertase subtilisin/kexin type 9; ox-LDL, oxidized low density lipoprotein; QUE, quercetin.
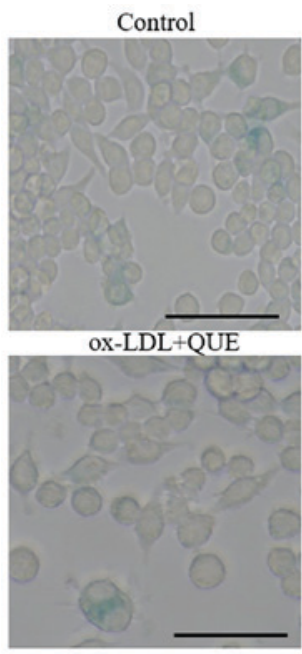
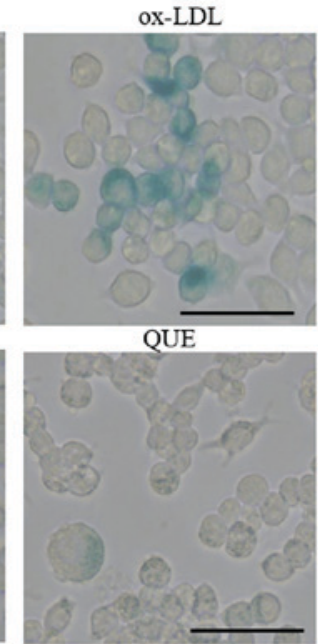

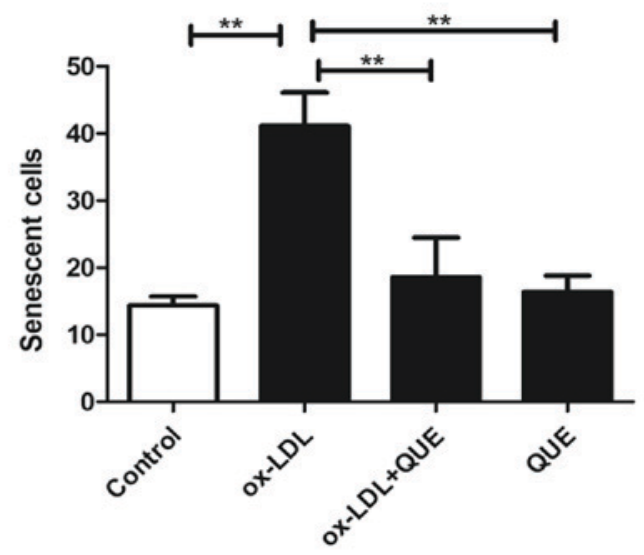

Figure 5. Quercetin and RAW 264.7 cell senescence measured using $\beta$-galactosidase staining. Data are presented as the mean \pm standard deviation. ${ }^{* *} \mathrm{P}<0.01$. Scale bar, $50 \mu \mathrm{m}$. ox-LDL, oxidized low density lipoprotein; QUE, quercetin.

leads to AS development (11). In early AS, mononuclear cells transform to macrophages which were induced by ox-LDL and inflammatory mediators in the endothelial gap. Then macrophages engulf ox-LDL to cause cholesterol ester accumulation and foam cell formation. Foam cells contribute to initial pathological changes leading to AS (3).

ABCA1 is an ABC transporter superfamily member mainly expressed in macrophages. ABCA1, as an integrated membrane protein, is necessary for lipid outflow transporters, and contributes to clearance of excess cholesterol via consumption of ATP and promotion of RCT $(12,13)$. As a major transporter of intracellular cholesterol efflux, ABCA1 promotes deposition of cholesterol in macrophages so it contributes to AS (14). ABCG1 is expressed in macrophages and ABCG1 expression contributes to RCT. ABCG1 expression can promote cholesterol outflow of macrophages, inhibit excessive cholesterol accumulation, and reduce the formation of foam cells (15). LXRs are cholesterol metabolic receptors that regulate expression of key genes in cholesterol metabolism, as well as participate in lipid metabolism, innate 
Table I. Cell SOD activity and MDA.

\begin{tabular}{lcc}
\hline Group & SOD $(\mu / \mathrm{ml})$ & MDA $(\mathrm{nmol} / \mathrm{ml})$ \\
\hline Control & $14.92 \pm 0.96$ & $3.73 \pm 0.03$ \\
ox-LDL & $12.54 \pm 0.89^{\mathrm{a}}$ & $2.31 \pm 0.08^{\mathrm{a}}$ \\
ox-LDL + QUE & $14.03 \pm 0.91^{\mathrm{b}}$ & $3.36 \pm 0.04^{\mathrm{b}}$ \\
QUE & $14.32 \pm 2.18^{\mathrm{c}}$ & $3.54 \pm 0.07^{\mathrm{c}}$ \\
\hline
\end{tabular}

Values are presented as the mean \pm standard error of the mean. ${ }^{\mathrm{a}} \mathrm{P}<0.01$ vs. control group. ${ }^{\mathrm{b}} \mathrm{P}<0.05,{ }^{\mathrm{c}} \mathrm{P}<0.01$ vs. ox-LDL group. SOD, superoxide dismutase; MDA, malondialdehyde; ox-LDL, oxidized low density lipoprotein; QUE, quercetin.

immune of macrophages, inflammatory response and other physiological activities (16). LXRs include two homologous subtypes, LXR- $\alpha$ and LXR- $\beta$. LXR- $\alpha$, a supranuclear receptor protein, is an oxidized cholesterol-activated nuclear receptor that regulates ABCA1 gene expression (17). PCSK9 is a subtilisin which regulates lipid metabolism (18). Studies of PCSK9 in AS are chiefly focused on regulation of lipid metabolism, and PCSK9 can promote degradation of low density lipoprotein receptor (LDLR) in hepatocytes and regulate lipid metabolism to change LDL-C (19). Moreover, PCSK9 can promote macrophage lipid accumulation and expression of inflammatory factors (20). Some studies show that PCSK9 inhibitors have anti-AS effects because they activate the LXR- $\alpha$ signaling pathway, and promote ABCA1 protein expression to accelerate cholesterol efflux (7). Studies indicate that P21 is involved in the regulation of cell proliferation, differentiation, migration, senescence and apoptosis. p21-mediated cell senescence may not be related to P53 (21). P 16 is a major inhibitor of cyclin dependent kinase (CDKK), and upregulation of P16 is key to cell senescence associated with cell growth arrest (22).

Quercetin may prevent AS (23) so it is often found in herbal products $(24,25)$ but these have not been studied for efficacy. Studies suggest that quercetin may reduce inflammatory factors and adhesion molecules of AS by regulating the TLR/NF- $\kappa \mathrm{B}$ signaling pathway (26) and upregulating ABCA1 proteins associated with $\mathrm{RCT}$ in $\mathrm{apoE}^{-/-}$mice as well as promoting cholesterol efflux (9). Furthermore, quercetin may activate the PPAR $\gamma$-ABCA1 pathway to promote cholesterol efflux in macrophages, thereby inhibiting the formation of foam cells (27). Quercetin may improve protein expression of ABCG1 in a concentration-dependent manner in vitro (9), which is consistent with in vivo data (28). Studies show that quercetin can inhibit lipopolysaccharide-induced RAW264.7 macrophages and downregulate the TLR4 signaling pathway to block inflammation (29). Quercetin also upregulates ABCA1 expression through p38 signaling pathway or activates the LXR- $\alpha$ signaling pathway in THP-1 cells to promote cholesterol efflux $(30,31)$. Studies suggest that quercetin can prevent apoptosis by regulating translational modification of P53 and P21 (32). In addition, studies indicate that quercetin may decrease expression of PCSK9 and increase expression of ABCA1 in hepatocytes of apoE $\mathrm{E}^{-/-}$mice (10). Thus, quercetin may block the development of AS.
We used $100 \mathrm{mg} / \mathrm{ml}$ ox-LDL to transform RAW264.7 cells to foam cells. Quercetin treatment improved cell viability of ox-LDL-induced RAW264.7 cells and blocked lipid accumulation. Furthermore, immunofluorescent and Western blot results showed that quercetin regulated expression of ABCA1, ABCG1, LXR- $\alpha$, PCSK9, P53, P21 and P16, to promote RCT in RAW264.7 cells, inhibit foam cell formation, and inhibit senescence. Finally, $\beta$-gal staining showed that quercetin could significantly reduce $\beta$-gal positive cells, and SOD and MDA data confirmed that quercetin reduced RAW264.7 cell aging. Chinese kidney-tonifying drugs are reported to regulate lipids in apoE $\mathrm{E}^{-/}$mice and CAS patients $(33,34)$, improving vascular elasticity and reducing senescence (35). These results above suggest that Chinese kidney-tonifying drugs may have a good therapeutic prospect for AS.

Thus, as one of the monomers of traditional Chinese medicine for tonifying the kidney, quercetin blocked damage of ox-LDL-induced RAW264.7 cells and improved viability, as well as reduced lipid accumulation and senescence. This may be attributed to effective regulation of ABCA1, ABCG1, LXR- $\alpha$, PCSK9, P53, P21 and P16 expression. Future studies will continue to study the effect of quercetin on the expression of related proteins in RCT.

\section{Acknowledgements}

Not applicable.

\section{Funding}

The present study was supported by grants from the Shanghai Nature Science Fund (grant no. 16ZR1433900), the Shanghai Health and Family Planning Commission Fund (grant no. 201640217) and Shanghai University of Traditional Chinese Medicine graduate 'innovation ability training' special research projects (grant no. Y201858).

\section{Availability of data and materials}

The datasets used or analysed during the current study are available from the corresponding author on reasonable request.

\section{Authors' contributions}

DS, SL, HC, SLX and CC conceived and designed the study. SL, HC and QJ performed the experiments. SL, QJ and HC made substantial contributions to the acquisition, analysis and interpretation of the data and wrote the paper. DS, CC and SLX reviewed and edited the manuscript. All authors read and approved the manuscript.

\section{Ethics approval and consent to participate}

Not applicable.

\section{Consent for publication}

Not applicable. 


\section{Competing interests}

The authors declare that they have no competing interests.

\section{References}

1. Getz GS and Reardon CA: The mutual interplay of lipid metabolism and the cells of the immune system in relation to atherosclerosis. Clin Lipidol 9: 657-671, 2014.

2. MaranhãoRC andLeite AC Jr:Development of anti-atherosclerosis therapy based on the inflammatory and proliferative aspects of the disease. Curr Pharm Des 21: 1196-1204, 2015.

3. Yu XH, Fu YC, Zhang DW, Yin K and Tang CK: Foam cells in atherosclerosis. Clin Chim Acta 424: 245-252, 2013.

4. Voloshyna I, Seshadri S, Anwar K, Littlefield MJ, Belilos E, Carsons SE and Reiss AB: Infliximab reverses suppression of cholesterol efflux proteins by TNF- $\alpha$ : A possible mechanism for modulation of atherogenesis. Biomed Res Int 2014: 312647, 2014

5. Cui Q, Ju X, Yang T, Zhang M, Tang W, Chen Q, Hu Y, Haas JV, Troutt JS, Pickard RT, et al: Serum PCSK9 is associated with multiple metabolic factors in a large Han Chinese population. Atherosclerosis 213: 632-636, 2010.

6. Paciullo F, Fallarino F, Bianconi V, Mannarino MR, Sahebkar A and Pirro M: PCSK9 at the crossroad of cholesterol metabolism and immune function during infections. J Cell Physiol 232: 2330-2338, 2017

7. Adorni MP, Cipollari E, Favari E, Zanotti I, Zimetti F, Corsini A, Ricci C, Bernini F and Ferri N: Inhibitory effect of PCSK9 on Abcal protein expression and cholesterol efflux in macrophages. Atherosclerosis 256: 1-6, 2017.

8. Lara-Guzman OJ, Tabares-Guevara JH, Leon-Varela YM, Álvarez RM, Roldan M, Sierra JA, Londoño-Londoño JA and Ramirez-Pineda JR: Proatherogenic macrophage activities are targeted by the flavonoid quercetin. J Pharmacol Exp Ther 343: 296-306, 2012.

9. Cui Y, Hou P, Li F, Liu Q, Qin S, Zhou G, Xu X, Si Y and Guo S: Quercetin improves macrophage reverse cholesterol transport in apolipoprotein E-deficient mice fed a high-fat diet. Lipids Health Dis 16: 9, 2017.

10. Mbikay M, Sirois F, Simoes S, Mayne J and Chrétien M: Quercetin-3-glucoside increases low-density lipoprotein receptor (LDLR) expression, attenuates proprotein convertase subtilisin/kexin 9 (PCSK9) secretion and stimulates LDL uptake by Huh7 human hepatocytes in culture. FEBS Open Bio 4 755-762, 2014

11. Lu Y and Jia Y: Quercetin upregulates ABCA1 expression through liver $\mathrm{X}$ receptor alpha signaling pathway in THP-1 macrophages. Eur Rev Med Pharmacol Sci 20: 3945-3952, 2016.

12. Yvan-Charvet L, Pagler T, Gautier EL, Avagyan S, Siry RL, Han S, Welch CL, Wang N, Randolph GJ, Snoeck HW and Tall AR: ATP-binding cassette transporters and HDL suppress hematopoietic stem cell proliferation. Science 328: 1689-1693, 2010.

13. Kolovou V, Marvaki A, Boutsikou M, Vasilopoulos G, Degiannis D, Marvaki C and Kolovou G: Effect of ATP-binding cassette transporter A1 (ABCA1) gene polymorphisms on plasma lipid variables and common demographic parameters in Greek nurses. Open Cardiovasc Med J 10: 233-239, 2016.

14. Wang S and Smith JD: ABCA1 and nascent HDL biogenesis. Biofactors 40: 547-554, 2014

15. Vaughan AM and Oram JF: ABCG1 redistributes cell cholesterol to domains removable by high density lipoprotein but not by lipid-depleted apolipoproteins. J Biol Chem 280: 30150-30157, 2005.

16. Oosterveer MH, Grefhorst A, Groen AK and Kuipers F: The liver X receptor: Control of cellular lipid homeostasis and beyond Implications for drug design. Prog Lipid Res 49: 343-352, 2010.

17. Ikhlef S, Berrougui $\mathrm{H}$, Kamtchueng $\mathrm{SO}$ and Khalil A: Paraoxonase 1-treated oxLDL promotes cholesterol efflux from macrophages by stimulating the PPAR $\gamma$-LXR $\alpha$-ABCA1 pathway. FEBS Lett 590: 1614-1629, 2016.

18. Sharma K and Baliga RR: Genetics of dyslipidemia and ischemic heart disease. Curr Cardiol Rep 19: 46, 2017.
19. Soutar AK: Unexpected roles for PCSK9 in lipid metabolism. Curr Opin Lipidol 22: 192-196, 2011.

20. Liu M, Wu G, Baysarowich J, Kavana M, Addona GH, Bierilo KK, Mudgett JS, Pavlovic G, Sitlani A, Renger JJ, et al: PCSK9 is not involved in the degradation of LDL receptors and BACE1 in the adult mouse brain. J Lipid Res 51: 2611-2618, 2010.

21. Romanov VS, Pospelov VA and Pospelova TV: Cyclin-dependent kinase inhibitor p21(Waf1): Contemporary view on its role in senescence and oncogenesis. Biochemistry (Mosc) 77: 575-584, 2012.

22. Shih CT, Chang YF, Chen YT, Ma CP, Chen HW, Yang CC, Lu JC, Tsai YS, Chen HC and Tan BC: The PPAR $\gamma$-SETD8 axis constitutes an epigenetic, p53-independent checkpoint on p21-mediated cellular senescence. Aging Cell 16: 797-813, 2017.

23. Zhang M, Xie Z, Gao W, Pu L, Wei J and Guo C: Quercetin regulates hepatic cholesterol metabolism by promoting cholesterol-to-bile acid conversion and cholesterol efflux in rats. Nutr Res 36: 271-279, 2016

24. Mehrbani M, Choopani R, Fekri A, Mehrabani M, Mosaddegh M and Mehrabani M: The efficacy of whey associated with dodder seed extract on moderate-to-severe atopic dermatitis in adults: A randomized, double-blind, placebo-controlled clinical trial. J Ethnopharmacol 172: 325-332, 2015.

25. Sun X, Yamasaki M, Katsube T and Shiwaku K: Effects of quercetin derivatives from mulberry leaves: Improved gene expression related hepatic lipid and glucose metabolism in short-term high-fat fed mice. Nutr Res Pract 9: 137-143, 2015

26. Bhaskar S, Sudhakaran PR and Helen A: Quercetin attenuates atherosclerotic inflammation and adhesion molecule expression by modulating TLR-NF- $\mathrm{B}$ B signaling pathway. Cell Immunol 310: 131-140, 2016.

27. Sun L, Li E, Wang F, Wang T, Qin Z, Niu S and Qiu C: Quercetin increases macrophage cholesterol efflux to inhibit foam cell formation through activating PPAR $\gamma$-ABCA1 pathway. Int J Clin Exp Pathol 8: 10854-10860, 2015.

28. Guo S, Tian H, Dong R, Yang N, Zhang Y, Yao S, Li Y, Zhou Y, Si Y and Qin S: Exogenous supplement of N-acetylneuraminic acid ameliorates atherosclerosis in apolipoprotein E-deficient mice. Atherosclerosis 251: 183-191, 2016.

29. Byun EB, Yang MS, Choi HG, Sung NY, Song DS, Sin SJ and Byun EH: Quercetin negatively regulates TLR4 signaling induced by lipopolysaccharide through Tollip expression. Biochem Biophys Res Commun 431: 698-705, 2013.

30. Chang YC, Lee TS and Chiang AN: Quercetin enhances ABCA1 expression and cholesterol efflux through a p38-dependent pathway in macrophages. J Lipid Res 53: 1840-1850, 2012.

31. Lu Y and Jia YP: Quercetin upregulates ABCA1 expression through liver $\mathrm{X}$ receptor alpha signaling pathway in THP-1 macrophages. Eur Rev Med Pharmacol Sci 20: 3945-3952, 2016

32. Gong C, Yang Z, Zhang L, Wang Y, Gong W and Liu Y: Quercetin suppresses DNA double-strand break repair and enhances the radiosensitivity of human ovarian cancer cells via p53-dependent endoplasmic reticulum stress pathway. Onco Targets Ther 11: $17-27,2017$

33. Shen DZ, Xing SL, Chen C, et al: Effect of shouqian granule on atherosclerosis in $\mathrm{ApoE}^{-/-}$mice based on expression of TLR4, MCP-1 and ICAM-1 in mice. Chin Med Emerg 2: 192-194+232, 2017.

34. Shen DZ, Chen C, Chen JL and Xing S: Effect of shoushen granules on level of blood lipids and inflammatory cytokines when treating carotid artherosclerosis. Chin Arch Tradit Chin Med 1: 22-24, 2014.

35. Shen DZ, Xing SL, Chen C, Shen R and Lou DF: Effect of Shoushen granule on arterial elasticity in patients with carotid atherosclerosis: a clinical randomized controlled trial. J Tradit Chin Med 4: 389-395, 2015.

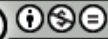

This work is licensed under a Creative Commons Attribution-NonCommercial-NoDerivatives 4.0 International (CC BY-NC-ND 4.0) License. 